\title{
Performance Analysis of FBG Temperature Sensors Based on Mach-Zehnder Interferometer
}

\author{
Shehab A. Kadhim ${ }^{a}$, Firas S. Mohammed ${ }^{b, *}$, \\ Ammar S. Alattar ${ }^{b}$ And Aseel I. Mahmood ${ }^{a}$
}

${ }^{a}$ Materials Research Directorate, Ministry of Science and Technology, Baghdad, Iraq

${ }^{b}$ Department of Physics, College of Sciences, Mustansiriyah, University, Baghdad, Iraq

Doi: $10.12693 /$ APhysPolA.140.316

*e-mail: fsphd@uomustansiriyah.edu.iq

\begin{abstract}
Advanced fiber optic sensing systems are in demand for different thermodynamics applications. In this article, an optical heterodyne Fiber Bragg Grating temperature sensors based on Mach-Zehnder interferometer are presented. The system uses a Fiber Bragg Grating as a tunable element from a single laser source. The experimental results show that the experimantal setup is stable while its costs and requirements are reduced by implementing a single source laser which generates two specific wavelengths $(1547.8 \mathrm{~nm}$ and $1550 \mathrm{~nm})$. The sensitivity of the modified system is about $15 \mathrm{pm} /{ }^{\circ} \mathrm{C}$, which is larger than other traditional detection systems. Efficient setting conditions, sensitivity and optimization of the correlation coefficient - these features are significant in detecting a synthetic heterodyne group because they are responsible for fast and sensitive topographic imaging.
\end{abstract}

topics: FBG sensors, Mach-Zehnder interferometer, heterodyne detection

\section{Introduction}

There is a considerable interest in the development of new optical fiber-based sensing devices. Such devices need to be reliable, highly sensitive, simple in fabrication and affordable to compete with conventional sensors. Recently, there is a rapid growth of technology of Fiber Bragg Gratings (FBG) as a temperature sensors [1-4]. Many different temperature sensing techniques are based on the structure of optical fibers, for example, Mach-Zehnder interferometers (MZI) [5-7], FabryPerot interferometers $[8,9]$ and Sagnac interferometer [10]. Parameters such as carrier heating influence semiconductor materials (optical fibers and FBG) [11]. The temperature sensitivity of FBGs dependeds on the refractive index modulation [12]. Furthermore, the fiber core refractive index $\Delta n$ varies according to [13]

$$
\Delta n(z)=\delta n\left[1+v \cos \left(\frac{2 \pi}{\Lambda} z\right)\right]
$$

where $\delta n$ is the average correction to the value of the fiber core refractive index, $v$ is the refractive index modulation coefficient $(0 \leq v \leq 1), \Lambda$ is the grating period, and $z$ is the fiber grating's axial location coordinate. The combination of the reference and sensing outputs produces a frequency shift, known as beat frequency $f_{b}$. The difference between the two signals can be represented as follows [14]

$$
f_{\mathrm{b}}=f_{\mathrm{s}}-f_{\mathrm{r}},
$$

where $f_{\mathrm{s}}$ and $f_{\mathrm{r}}$ are sensing and reference frequencies. The signal intensity of the electro-optical detector can be described as follows [15]

$$
\begin{aligned}
I & =\frac{1}{2} A_{\mathrm{s}}^{2}\left[1+\cos \left(2 \omega_{\mathrm{s}} t+2 \theta_{1}\right)\right] \\
& +\frac{1}{2} A_{\mathrm{r}}^{2}\left[1+\cos \left(2 \omega_{r} t+2 \theta_{2}\right)\right] \\
& +A_{\mathrm{s}} A_{\mathrm{r}} \cos \left(\left(\omega_{\mathrm{s}}+\omega_{\mathrm{r}}\right) t+\theta\right) \\
& +A_{\mathrm{s}} A_{\mathrm{r}} \cos \left(\left(\omega_{\mathrm{s}}-\omega_{\mathrm{r}}\right) t+\theta\right)
\end{aligned}
$$

where $A_{\mathrm{s}}$ and $A_{\mathrm{r}}$ are the amplitudes in the sensing and reference arms, $\omega_{\mathrm{s}}$ and $\omega_{\mathrm{r}}$ are the angular frequencies in the sensing and reference arms, respectively. The angle of light incidence is denoted by $\theta$, while lower indexes 1,2 correspond to the sensing and reference light waves, respectively. We used FBG to get a tunable source in a simulation of a modified heterodyne optical system [16]. In this work, a proposed standard and a modified optical heterodyne FBG temperature sensor based on MZI technique were investigated.

\section{Experimental setup}

Our experiment described in this paper is devided into two parts. Let us start with the block diagram of the standard optical heterodyne FBG temperature sensor based on MZI technique, illustrated 


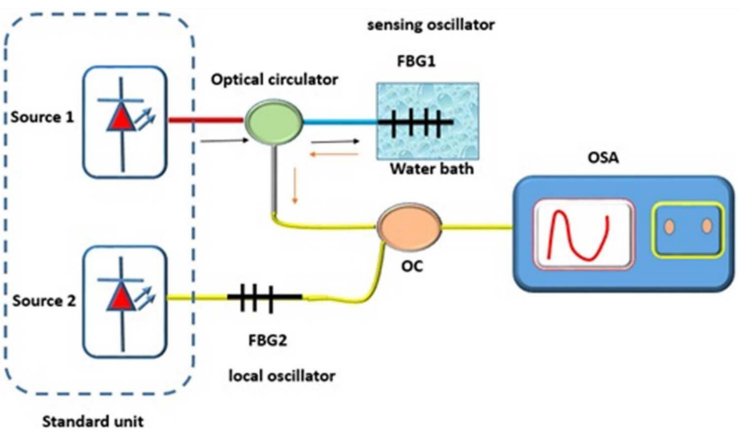

Fig. 1. Block diagram of standard optical heterodyne FBG temperature sensor based on MZI technique.

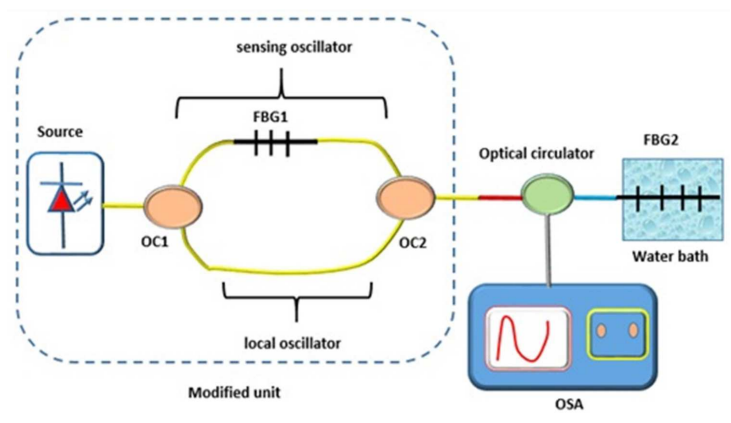

Fig. 2. Block diagram of modified optical heterodyne FBG temperature sensor.

in Fig. 1. The MZI design consists of two arms, i.e., the sensing and the reference arm. The sensing arm consists of (i) a single-mode laser source 1 "fiber pigtailed diode (Thorlabs)" with wavelength of $1550 \mathrm{~nm}$ and power of $1.5 \mathrm{~mW}$, connected to the red fiber (input port) of the optical fiber circulator, (ii) FBG1 (with Bragg wavelength equal to 1548.254 $\mathrm{nm}$, reflectivity $89 \%$, and bandwidth $0.183 \mathrm{~nm}$ ) which is immersed in a controlled temperature water bath. The input port of FBG1 is connected to the blue fiber of the optical fiber circulator and thus the reflected wave of FBG1 travels along the white fiber (output port) to be detected. The reference arm consists of another laser source 2 coupled with a fiber (ONTI inc) of wavelength $1550 \mathrm{~nm}$ and maximum power $3 \mathrm{~mW}$. These two sources are slightly different in wavelenght to achieve the heterodyne condition. The input port of the laser is connected to the input of the FBG2 which has a broad Bragg wavelength range $1530-1573 \mathrm{~nm}$ with $\pm 0.3 \mathrm{~nm}$, reflectivity $95 \pm 5 \%$, bandwidth $0.1-2.0 \mathrm{~nm}$. The two outputs of the MZI are joined by an optical coupler. Then, the output port is connected to OSA to record the spectra for each applied temperature. The applied temperature is $30-60^{\circ} \mathrm{C}$ with the step value of $5^{\circ} \mathrm{C}$.

Moreover, the block diagram of the modified optical heterodyne FBG temperature sensor based on the MZI interferometer is shown in Fig. 2. Here, the modification in FBG is achieved by using one optical source instead of two sources - just as in the second setup. The heterodyne system will be generated with a single-mode laser source "fiber pigtailed diode (Thorlabs)" with a wavelength of $1550 \mathrm{~nm}$, and power $1.5 \mathrm{~mW}$ connected to the optical coupler (operating wavelength 1280-1620 nm , operating temperature $30-60^{\circ} \mathrm{C}$ and coupling ratio $50 \%$ ). The first output port connects to FBG1 with the output wavelength $1547.8 \mathrm{~nm}$, different from the original wavelength. The second output port represents the second source with the wavelength $1550 \mathrm{~nm}$. Thus, we get two sources from one source with a slight difference between their wavelengths as desired for the heterodyne condition.

\section{Results and discussion}

In order to measure the response of the sensing systems, first we record the spectra of laser sources and the FBG sensor and reference, as shown in Fig. 3. One can notice that there is a shift towards the infrared (IR) region due to the Bragg wavelength condition.

The results of the two waves interference causing an intensity modulation at the beat "frequency" (heterodyne condition) are given by (3). The heterodyne spectra for the two used laser sources, the standard heterodyne, and the modified heterodyne systems are shown in Fig. $4 \mathrm{a}-\mathrm{c}$, respectively.

The spectra of the reflected FBG wavelengths was recorded and analyzed for each sensing temperature for different temperature sensor configurations, as shows in Fig. 5. The reflection spectra collected
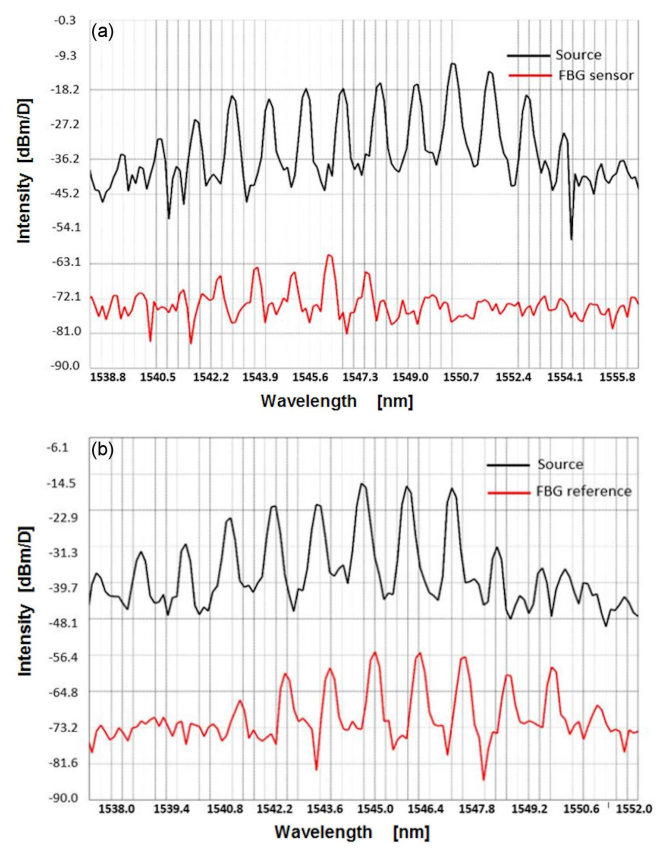

Fig. 3. The laser of (a) FBG sensor, (b) FBG reference spectra. 

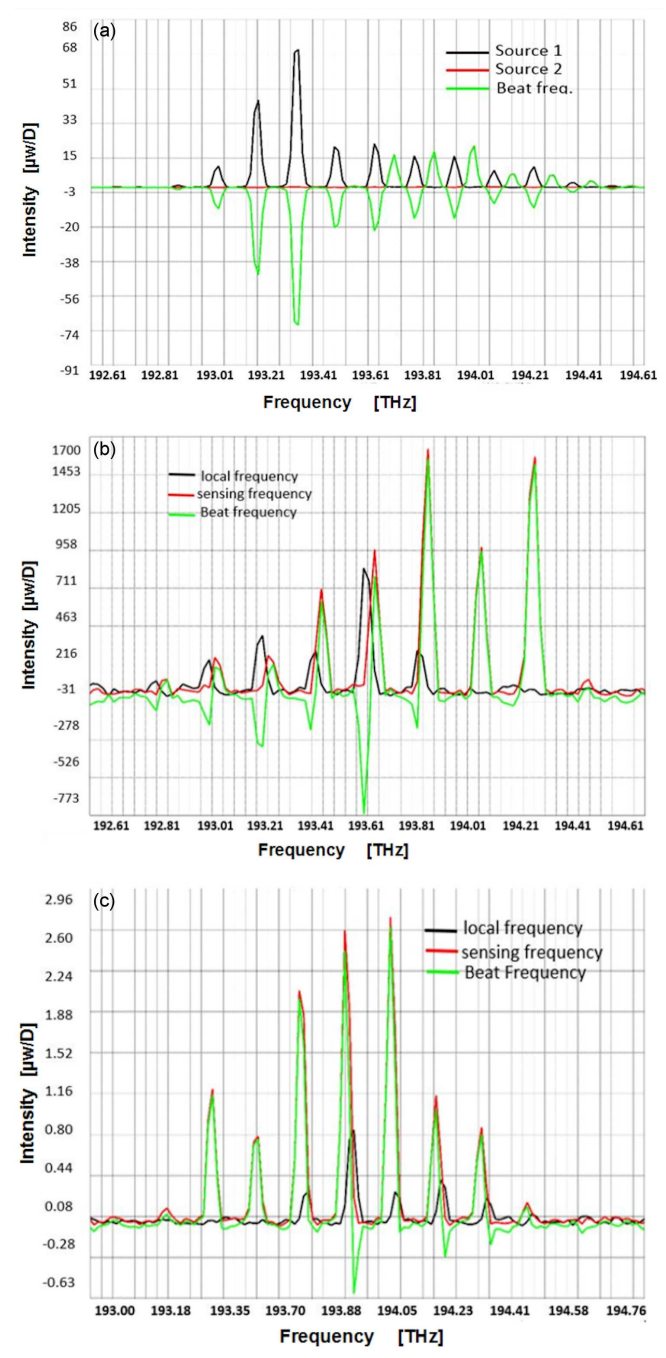

Fig. 4. The beat frequency of heterodyne detection using SMF's at room temperature for (a) two light sources, (b) standard system, and (c) modified system.

online are based on (a) reflected spectra of FBG, (b) standard MZI and heterodyne system, (c) modified MZI and heterodyne system, respectively. The temperature was increased from 30 to $60^{\circ} \mathrm{C}$, mostly in steps of $5^{\circ} \mathrm{C}$.

In Fig. 5, we can notice that the temperature variation leads to shifting in the Bragg wavelength towards longer wavelengths. The intensity decreases when the temperature of the FBG sensor increases. Moreover, for heterodyne systems based on two sources and one source, an intensity fluctuation can be observed. This can be a result of temperature variation or of optical power losses due to imperfect connection between the optical coupler and circulator. In addition, the indication of sensitivity of the temperature sensor is related to the Bragg wavelength shift, not to the intensity variation. The relation between the shifted Bragg wavelength and the applied temperature of each experiment are shown in Fig. 6.
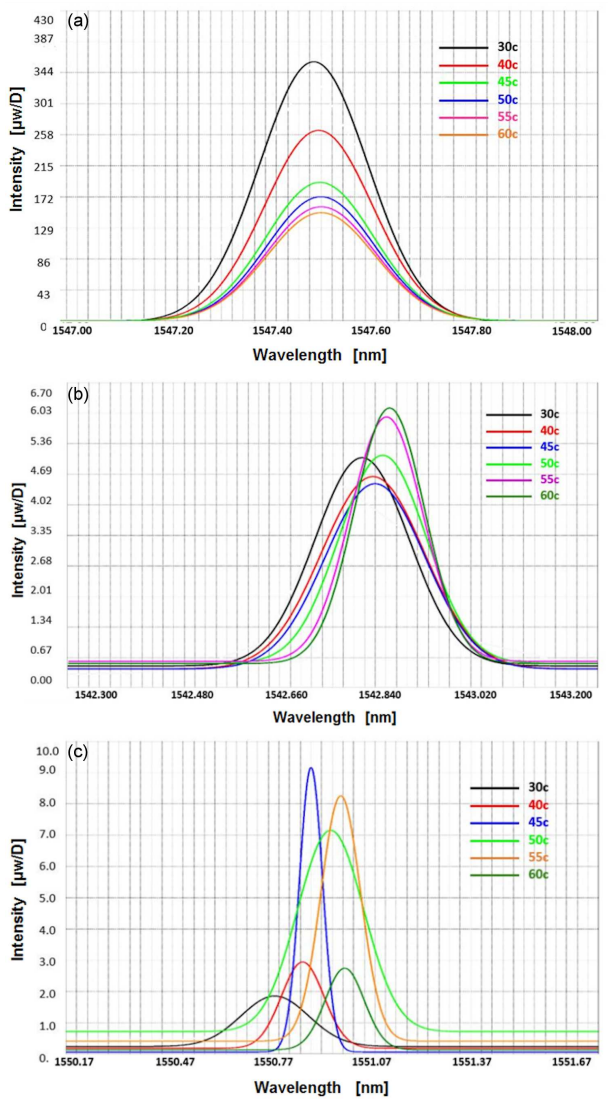

Fig. 5. The reflected spectra collected online for different temperature sensor schematics based on, (a) standard heterodyne system based on MZI technique, (b) modified heterodyne system.

The sensitivity was calculated from the slope of the linear relationship in Fig. 6, and it equal to $7 \mathrm{pm} /{ }^{\circ} \mathrm{C}, 9 \mathrm{pm} /{ }^{\circ} \mathrm{C}$, and $15 \mathrm{pm} /{ }^{\circ} \mathrm{C}$ with the correlation factor equal to $0.99,0.98$, and 0.99 for the temperature sensor based on the reflected spectra of FBG, standard MZI and heterodyne system, and modified heterodyne system, respectively. From the above results, the modification in the optimized system leads to the enhancement of sensor sensitivity. Our results agree with [16] where couple of FBGs was implemented.

\section{Conclusions}

In this work, a tunable source from a single laser source is obtained, by utilizing a proposed design of a heterodyne optical system. The use of a single source laser to generate two distinct wavelengths provides performance stability while also lowering costs and other requirements. The sensitivity of the proposed system is about $15 \mathrm{pm} /{ }^{\circ} \mathrm{C}$ with correlation factor equal to 0.99. Efficient setting conditions, sensitivity and optimization of the correlation coefficient - these features are significant in detecting a synthetic heterodyne group because they are responsible for fast and sensitive topographic imaging. 

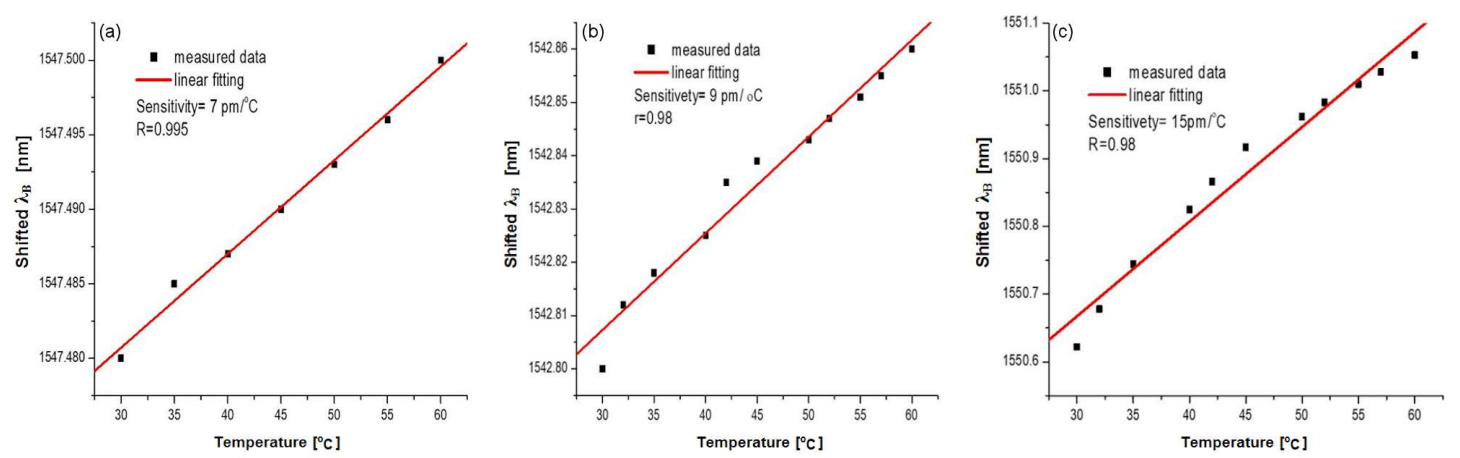

Fig. 6. The relationship between shifted Bragg wavelength and applied temperature for (a) reflected spectra of FBG, (b) standard heterodyne system based on MZI technique, (c) modified MZI heterodyne system.

\section{Acknowledgments}

The authors would like to thank Mustansiriyah university (www. uomustansiriyah.edu.iq) Baghdad-Iraq for its support in the present work.

\section{References}

[1] N.F. Naim, S.N.M. Sudin, S.S. Sarnin, N. Ya'acob, L.S. Supian, Int. J. Electr. Comput. Eng. 10, 3158 (2020).

[2] H.M. El-Gammal, E.A. El-Badawy, M.R.M. Rizk, M. H. Aly, Opt. Quantum Electron. 52, 2171 (2020).

[3] M.D. Shree, A. Sangeetha, P. Krishnan, Plasmonics 15, 813 (2020).

[4] F. Esposito, A. Srivastava, A. Iadicicco, S. Campopiano, Opt. Laser Technol. 113, 198 (2019).

[5] F. Zhang, X. Xu, J. He, B. Du, Y. Wang, Opt. Lett. 44, 2466 (2019).

[6] S.-N. Wang, R.-Q. Lv, Y. Zhao, J.-K. Qian, Opt. Fiber Technol. 45, 93 (2018).

[7] T. Liu, J. Wang, Y. Liao, X. Wang, S. Wang, Opt. Express 26, 12277 (2018).
[8] X.L. Cui, H. Zhang, D.N. Wang, Opt. Lett. 45, 726 (2020).

[9] G. Zhang, X. Wu, S. Li, W. Liu, S. Fang, C. Zuo, W. Zhang, B. Yu, Appl. Opt. 59, 873 (2020).

[10] L. Liu, H. Gao, T. Ning, L. Pei, J. Zheng, J. Li, J. Xu, Opt. Laser Technol. 123, 105951 (2020).

[11] A.H. Flayyih, F.S. Mohammed, A.H. AlKhursan, Microw. Opt. Technol. Lett. 63, 2231 (2020).

[12] C. Martelli, J. Canning, N. Groothoff, K. Lyytikainen, Opt. Lett. 30, 1785 (2005).

[13] H. Qiu, F. Min, Y. Yang, Adv. Aerodyn. 2, 10 (2020).

[14] L. Huanhuan, F. Pang, L. Lv, X. Mei, Y. Song, J. Chen, T. Wang, IEEE Photonics J. 10, 1 (2018).

[15] J.M. Senior, M.Y. Jamro, Optical Fiber Communications Principles and Practice, 3rd ed, Pearson Education Limited, 2009.

[16] Q. Zhao, H.K. Zheng, R.Q. Lv, F.Y. Gu, Y. Zhao, Y. Yang, Sens. Actuators A 280, 68 (2018). 